\title{
Thermal Injuries during Intraoperative Magnetic Resonance Imaging: Mechanisms and Prevention
}

\author{
Nitin Manohar ${ }^{1}$ Astha Palan ${ }^{1}$ Harsh Deora ${ }^{2}$ Boyina J. Rajesh ${ }^{3}$ Anand Balasubramanium ${ }^{3}$ \\ Kambaduru Pradeep ${ }^{1}$ \\ ${ }^{1}$ Department of Neuroanesthesia, Yashoda Hospitals, \\ Secunderabad, Telangana, India \\ 2Department of Neurosurgery, Sanjay Gandhi Post Graduate \\ Institute of Medical Sciences, Lucknow, Uttar Pradesh, India \\ ${ }^{3}$ Department of Neurosurgery, Yashoda Hospitals, Secunderabad, \\ Telangana, India \\ J Neuroanaesthesiol Crit Care:2021;8:66-68

\begin{abstract}
Address for Correspondence Harsh Deora, MS, MCh, DNB, Department of Neurosurgery, Sanjay Gandhi Post Graduate (e-mail: demo5601@gmail.com).
\end{abstract} \\ Institute of Medical Sciences, Lucknow 226014, Uttar Pradesh, India
}

\begin{abstract}
Keywords

- intraoperative magnetic resonance imaging

- neurosurgery

- thermal burns

Background Thermal injuries in a patient undergoing magnetic resonance imaging (MRI) are rare; more so, when the patient in question is being operated upon. We attempt to elucidate the various factors that may predispose to such an unfortunate circumstance, through a series of four cases.

Materials and Methods We conducted a retrospective review of our experience with intraoperative MRI and found four cases of burns attributed to MRI. Factors leading to possible injury were examined after other causes were ruled out.

Results Collection of moisture between the leads and the patient's skin was the most common factor for the burns. There were no instances of closed loop formation or injury due to direct contact of cables to the skin.

Conclusion Awareness of the causative factor can lead to prevention. Proper education of all concerned personnel involved in the conduction of the intraoperative MRI is paramount to prevention of the same.
\end{abstract}

\section{Introduction}

Thermal injuries in magnetic resonance imaging (MRI) scanners are known to be rare complications. Here we present four cases in which we encountered thermal injuries during intraoperative 3 Tesla MRI under general anesthesia for neurosurgeries. We also discuss possible mechanisms causing thermal burn injuries in intraoperative MRI (IOMRI) and suggest ways to reduce them.

The etiology of MRI-induced thermal injury has not been completely explained and may be multifactorial. MRI systems require the use of radiofrequency (RF) pulses to create the magnetic resonance signal. This RF energy is transmitted through free space from the RF coil to the patient. When conducting materials are placed within this RF field, a concentration of electrical currents sufficient to cause excessive heating can be produced and tissue damage may occur. ${ }^{1}$ Most burn injuries reported till now occurred where

Published online May 2, 2020
DOI https://doi.org/

10.1055/s-0039-1685247 ISSN 2348-0548.
The mechanism, which has been considered responsible for heating of the coils eventually leading to burns, is the direct electromagnetic induction. Other mechanisms producing heat in the MRI environment are heating in a resonant circuit and heating due to the antenna effect. ${ }^{2-4}$

\section{Materials and Methods}

We collected a record of all cases that had burns while using a 3-Tesla MRI suite-operating theater over a period of 1 year, with 150 cases studied from October 2017 to October 2018. There were four such cases noted. Two cases were operated upon in the lateral position and one each in prone and supine. The patient was moved in between surgery with sterile drapes into the adjacent MRI suite for scan. IOMRI for neurosurgeries can pose a higher risk of thermal injuries as the surgeries are long in duration during which the patient is covered under drapes, so there may be collection of sweat 
or moisture in areas where coil touches skin in the body. This can induce thermal injuries due to excess heat production during an IOMRI scan. Unfortunately, these can be detected only after the end of surgery.

\section{Results}

All cases were neurosurgical cases performed under general anesthesia, two cerebellopontine angle lesions, one parietooccipital glioma, and one pituitary adenoma. All patients were monitored using the MRI-compatible multi-parameter monitor (Tesla, Germany) during the IOMRI scan. A complete institutional checklist was performed to ensure patient, personnel, and equipment safety before moving in.

\section{Case 1}

Right parieto-occipital glioma operated in the prone position postoperatively had a superficial burn on the nose (-Fig. 1A). We hypothesized that this was due to direct contact of nose with the lower MRI coil or moisture collected between the lower coil and nose in prone position. Therefore, after this case, we made sure that the lower coil is placed after surgery

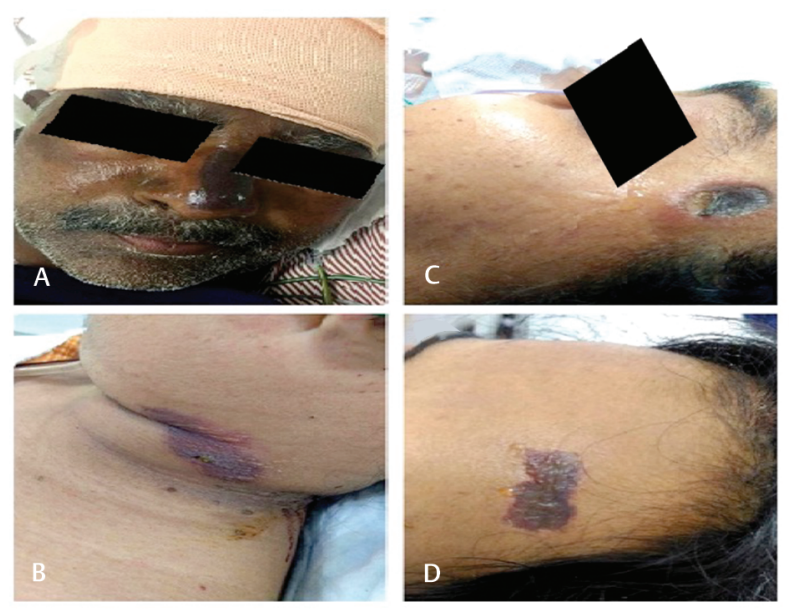

Fig. 1 (A) Superficial burn on the nose in prone position. (B) Thermal injury between skin folds in the flexed area of neck. (C) Superficial burn under the navigation tracker. (D) Thermal injury under area of BIS sensor on the forehead. BIS, bispectral index. just before moving in for the IOMRI scan so that moisture is not collected at the dependent sites.

\section{Case 2}

Cerebellopontine angle tumor operated in left lateral position with neck rotation and lateral flexion had superficial thermal injury in flexed neck region postoperatively (-Fig. 1B). We postulated that this could be due to collection of moisture or sweat in flexed neck area leading to heat generation causing burn inside the magnetic field.

\section{Case 3}

Pituitary macroadenoma for transnasal transsphenoidal resection with the use of neuronavigation had a superficial burn postoperatively under the MRI-compatible navigation tracker site or reference coil (StealthStation EM noninvasive patient tracker; Medtronic-Sofamor Danek, United States) ( - Fig. 1C). We hypothesized that this could be due to improper contact between the navigation tracker and patient's skin, allowing moisture to be collected. Because the navigation tracker ideally needs to be discarded once the glue attaching it to the skin wears off, we have stopped using the same tracker for multiple cases and discard the same sooner. This prevents such burn incidents and ensures proper navigation accuracy.

\section{Case 4}

Cerebellopontine angle tumor operated with bispectral index (BIS) monitoring had a superficial burn on the forehead, in the area of BIS sensor, which was removed prior to the IOMRI scan (-Fig. 1D). Our hypothesis was that this burn would be due to the moisture collected under the BIS sensor due to prolonged duration of surgery. After this case, we started cleaning and drying the site of BIS sensor before going for IOMRI.

All cases had a dermatology consultation, and since all of them were grade I burns, they healed completely without sequelae or scarring with just bacitracin and silver sulfadiazine dressings.

\section{Discussion}

A review of the areas burned in these cases clearly brings out the causative mechanism (-Table $\mathbf{1}$ ).

Table 1 Review of the burned areas along with mechanisms of prevention and duration of surgery

\begin{tabular}{|c|c|c|c|c|}
\hline Cases & Mechanism & Region of burn & Prevention & Surgery and duration (surgery + MRI) \\
\hline 1 and 2 & $\begin{array}{l}\text { Most-dependent } \\
\text { portion of the } \\
\text { patient in contact } \\
\text { with MRI coils }\end{array}$ & $\begin{array}{l}\text { Nose (prone) } \\
\text { Neck (lateral) }\end{array}$ & $\begin{array}{l}\text { Place coil just before } \\
\text { shifting to the MRI } \\
\text { suite }\end{array}$ & $\begin{array}{l}\text { Case 1: right parieto-occipital craniotomy and } \\
\text { excision } \\
\text { Duration: } 8 \mathrm{~h}(7+1) \\
\text { Case } 2: \text { retromastoid suboccipital craniotomy and } \\
\text { excision of vestibular schwannoma } \\
\text { Duration: } 14 \mathrm{~h}(13+1)\end{array}$ \\
\hline 3 and 4 & $\begin{array}{l}\text { Glued electrodes/ } \\
\text { trackers have } \\
\text { moisture }\end{array}$ & $\begin{array}{l}\text { Fore head } \\
\text { (navigation/BIS } \\
\text { electrodes) }\end{array}$ & $\begin{array}{l}\text { 1. Remove and clean } \\
\text { the BIS sensor/ } \\
\text { tracker } \\
\text { 2. Use only till the } \\
\text { glue perfectly sticks }\end{array}$ & $\begin{array}{l}\text { Case 3: endoscopic endonasal transsphenoidal } \\
\text { decompression of pituitary adenoma } \\
\text { Duration: } 10 \mathrm{~h}(9+1) \\
\text { Case 4: retromastoid suboccipital craniotomy and } \\
\text { excision of vestibular schwannoma } \\
\text { Duration: } 14 \mathrm{~h}(13+1)\end{array}$ \\
\hline
\end{tabular}

Abbreviations: BIS, bispectral index; MRI, magnetic resonance imaging. 


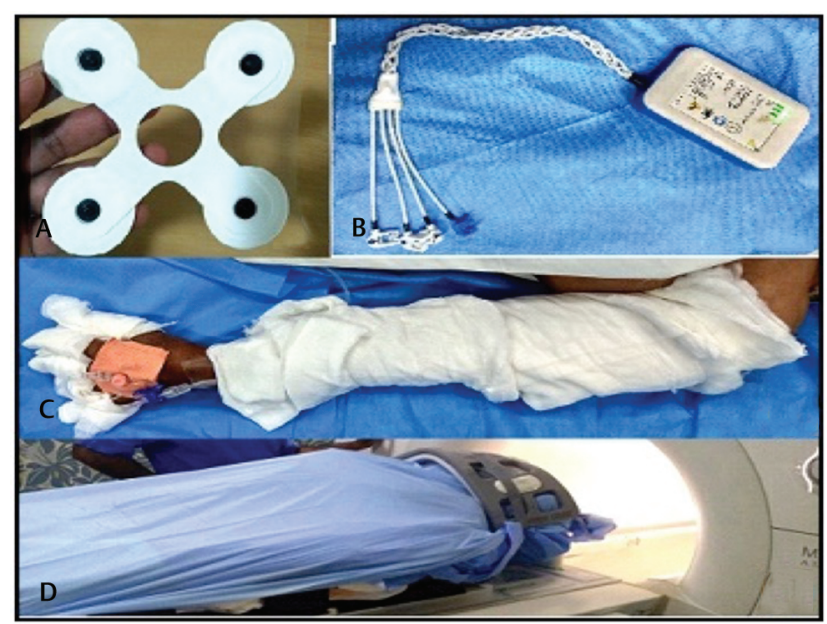

Fig. 2 (A) Closely placed MRI-compatible ECG leads made of carbon. (B) Braided MRI-compatible ECG wires with short connection ends to prevent looping. (C) Packing in areas of skin-to-skin contact (finger webs and axilla). (D) Patient under the drapes going into intraoperative MRI with upper coil visible. ECG, electrocardiogram; MRI, magnetic resonance imaging.

Because electrocardiographic (ECG) electrodes were removed while shifting the cases to the MRI room, none of the patients had any burns over the chest region where these were placed. Again, because new ECG electrodes were used in every single case, there were no burns, thus proving our hypothesis. Since application of these measures from October 2018, we have not had any similar burns cases in the further 50 cases conducted since then.

Based on our experience with intraoperative MRI (> 200 cases), we suggest following measures to prevent these thermal mishaps during surgeries. ${ }^{5}$

We should only use monitoring devices that are cleared for use with MRI during scanning. Placement of ECG leads ( - Fig. 2A) should be as close as possible, and wires should be braided and cables should be of short length ( - Fig. 2B). All areas that are likely to experience thermal burn injuries should be padded for thermal insulation (e.g., areas of skin-to-skin contact such as fingers, toes, axilla, and groin or skin-to-machine contact) ( - Fig. 2C, D).

There are many factors that may cause thermal injuries in intraoperative MRI-guided injuries. ECG cables should not form closed loops as they can pick up radiofrequency signals and heat up. ${ }^{6}$ All monitoring cables should be braided, not form loops and covered with padding to provide insulation. There should be no collection of moisture between skin folds and minimal soiling with surgical fluids. At the end of the surgery, all patients should be thoroughly checked for any thermal injury.

There has been only one other series of 57 cases with a total of 1,093 electrodes placed who underwent surgery in an IOMRI setting. In this prospective trial only 1 case had Grade I burns (1.75\%) which co-relates well with our series. ${ }^{7}$ With further experience and knowledge about the mechanisms, we should be able to eliminate any such cases in the future.

\section{Conclusion}

Most thermal injuries caused by MRI occur as a result of direct contact with the MRI coil, equipment, cables or wires, improper patient positioning, or due to collection of moisture under the drapes. Increased awareness among OT personnel, knowledge of mechanisms, institute-based checklist, and protocols can minimize these thermal injuries.

\section{Conflict of Interest}

None declared.

\section{References}

1 Hardy PT II, Weil KM. A review of thermal MR injuries. Radiol Technol 2010;81(6):606-609

2 Dempsey MF, Condon B. Thermal injuries associated with MRI. Clin Radiol 2001;56(6):457-465

3 Dempsey MF, Condon B, Hadley DM. Investigation of the factors responsible for burns during MRI. J Magn Reson Imaging 2001;13(4):627-631

4 Abdel-Rehim S, Bagirathan S, Al-Benna S, O'Boyle C. Burns from ECG leads in an MRI scanner: case series and discussion of mechanisms. Ann Burns Fire Disasters 2014;27(4):215-218

5 Shellock FG, Crues JV. MR procedures: biologic effects, safety, and patient care. Radiology 2004;232(3):635-652

6 Gandhe RU, Bhave CP. Intraoperative magnetic resonance imaging for neurosurgery-an anaesthesiologist's challenge. Indian J Anaesth 2018;62(6):411-417

7 Sarnthein J, Lüchinger R, Piccirelli M, Regli L, Bozinov O. Prevalence of complications in intraoperative magnetic resonance imaging combined with neurophysiologic monitoring. World Neurosurg 2016;93:168-174 